\title{
El acoso escolar en estudiantes con necesidades educativas especiales en una zona rural del Ecuador
}

Bullying on students with special educational needs in a rural area of Ecuador

\author{
Rosa $\mathbf{M}^{\mathrm{a}}$ Espada \\ e-mail: rosa.espada@urjc.es \\ Universidad Rey Juan Carlos. España \\ Sergio Sánchez (iD) \\ e-mail: sergio.sanchezfuentes@uam.es \\ Universidad Autónoma de Madrid. España \\ Rosa A. Sánchez \\ e-mail: rosysan16@hotmail.com \\ Unidad Educativa Dr. José María Velasco Ibarra. El Ecuador
}

\section{Resumen}

El acoso escolar es uno de los principales retos a los que se enfrentan los sistemas educativos modernos. Especialmente relevante es esta cuestión cuando las personas con necesidades educativas especiales sufren dicho acoso. Este trabajo tiene como objetivo conocer la prevalencia del acoso escolar en estudiantes con necesidades educativas especiales en un entorno rural. Para ello, un total de 115 estudiantes, junto con 8 docentes, participaron en el estudio. Dos cuestionarios ad hoc fueron creados para tal fin. Los resultados muestran que los estudiantes con discapacidad intelectual y del desarrollo y los estudiantes con autismo sufren en mayor medida situaciones de acoso escolar. Sin embargo, los estudiantes con trastornos del aprendizaje son el colectivo, dentro de los estudiantes con necesidades educativas especiales, que menos porcentaje de situaciones de acoso reportaron. En cuanto a las condiciones en las que se desarrollan estas situaciones entre centros públicos y particulares, los datos muestran como en los centros particulares no existe formación al respecto, se comunica en menor medida estas situaciones a las familias y, en líneas generales, se intenta ocultar el problema. Los autores concluyen que existe una necesidad incipiente de trabajar estos temas en las aulas y con el profesorado de todo tipo de centros educativos.

Palabras clave: acoso escolar; necesidades educativas especiales; discapacidad, educación .

\section{Abstract}

Bullying is one of the main challenges faced by modern education systems. This question is especially relevant when people with special educational needs suffer such harassment. This paper aims to know the prevalence of bullying on students with special educational needs in a rural environment. For this, a total of 115 students, along with 8 teachers participated in the study. Two ad hoc questionnaires were created for this purpose. The results show that students with intellectual and developmental disabilities and students with autism experience bullying situations to a greater extent. However, students with learning disabilities are the group, among students with special educational needs, that reported the lowest percentage of bullying situations. Regarding the conditions in which these situations develop between public and private centers, the data show that in private centers there is no training in this regard, these situations are communicated to a lesser extent to families and, in general, an attempt is made to hide the problem. The authors conclude that there is an incipient need to work on these issues in classrooms and with teachers in all types of educational centers.

Keywords: bullying; special education needs; disability; education.

Cómo referenciar este artículo / How to reference this article:

Espada, R. M., Sánchez, S. \& Sánchez, R. (2020). El acoso escolar en estudiantes con necesidades educativas especiales en una zona rural de El Ecuador. Tendencias Pedagógicas, 37, pp. 31-42. doi: 10.15366/tp2021.37.004 


\section{Introducción}

El acoso escolar, o también denominado bullying, es un tema prioritario en los sistemas educativos actuales (Lugones \& Ramírez, 2017). El acoso, frente a otras formas de violencia, se presenta con mayor frecuencia en los centros educativos entre iguales, independientemente de la procedencia, la ubicación de la escuela, el tipo de centro o los recursos del entorno (Vargas \& Paternina, 2017). Generalmente, comienza con ofensas de forma verbal, sometimientos intimidantes, mensajes de advertencias y marginación del grupo, pasando por diferentes estadios hasta que, en un alto porcentaje de casos, esta situación deriva en agresión física (Abadio et al., 2015).

De acuerdo con diversos autores, el bullying se presenta en dos tipos. El primero se refiere al acoso físico, siendo la segunda modalidad un acoso más centrado en aspectos psicológicos focalizados en el rechazo, la humillación o el aislamiento que hacen que se perjudiquen sus relaciones con el entorno y los iguales (Enríquez \& Garzón, 2015). Además, en la actualidad cada vez con más frecuencia también se encuentra otro tipo de acoso escolar. Este es el denominado cyberbullying, definido en el mismo contexto que el acoso tradicional, pero perpetrado a través de recursos electrónicos, como teléfonos o redes sociales, internet, apps, etc. (Herrera, Romera, \& Ortega, 2018). De hecho, este tipo de acoso está aumentando de manera exponencial, con lo que ya se está demandando por parte de la comunidad científica una intervención de manera inmediata (Garaigordobil, 2015; Gómez, 2014; Arias, 2014).

Las formas de acoso en la escuela requieren de medios para la identificación (Vergel, Martínez y Zafra, 2016) ya que este conlleva conducta agresiva y violenta en un ambiente educativo entre pares en el caso del acoso físico, o de persecución y acoso cibernético si se trata del ciberacoso. Ambos modelos se fundamentan desde los criterios básicos que describe Abadio et al., (2015): el acoso debe ser deliberado, reiterado y debe existir una situación de dominio y sumisión. Esto se concreta en bloqueo social, que se refiere al aislamiento total de la víctima, es decir, lo excluyen de todas las actividades sociales o educativas; hostigamiento, que hace referencia al maltrato constante ya sea físico, verbal o psicológico por un lapso de tiempo determinado; manipulación, donde su objetivo es burlarse y criticar delante de los pares para ponerlos en ridículo haciendo que todos actúen en contra de quienes sufren el acoso; y coacciones, donde los agresores buscan dominar la voluntad de sus víctimas para obtener lo que quieren (Lugones \& Ramírez, 2017).

En base a la definición del bullying, esta acción de violencia que se observa comúnmente en los centros escolares, ya sea entre iguales o de condiciones diferentes, intervienen tres actores: agresor, víctima y espectador, los mismos que se identifican por comportamientos irregulares en donde el o los agresores identifican la vulnerabilidad de la víctima y la atacan y convierten al espectador en un encubridor activo o sigiloso quien calla lo sucedido (Aristimuño \& Noya, 2015; Cobián, Nizama, Ramos, \& Mayta, 2015; Ruiz, Riuró, \& Tesouro, 2015).

En la dinámica del bullying, el agresor escoge a su víctima preferentemente con características de inseguridad, de condición física débil, de otras etnias o razas, con limitaciones en sus capacidades (Erazo, 2016; Córdova, Velázquez, \& Silva, 2015). Esto se hace complejo cuando los entornos son espacios rurales donde estas cuestiones tienden a ser más herméticas, se suele tener un sentimiento de vergüenza más integrado y donde las relaciones cercanas entre todos los miembros de la comunidad hacen que sea más complicado señalar e identificar ciertos roles que pueden suponer una estigmatización de la persona que lo sufre o de la persona que lo realiza.

Dentro de las principales consecuencias emocionales del bullying que se presentan en estudiantes se pueden mencionar algunas como: tensión, se presentan por el temor que posee la víctima de encontrarse con el o los agresores; autoestima baja, etc. (Asanza, Pilco, \& Alvarado, 2015). Cuestión que se vuelve a reforzar en la afirmación descrita en el párrafo anterior. Además, diversos trabajos ya vienen alertando sobre la especial vulnerabilidad de los niños con necesidades educativas especiales (de ahora en adelante NEE) (Moreno-Rodríguez et al., 2017) donde las consecuencias son más graves porque se encuentran en situaciones de mayor desprotección, no actúan en defensa propia en todos los casos y en muchas ocasiones prefieren callar las agresiones (Amaya, 2016; Garaigordobil, Martínez, \& Páez, 2015; García \& Ascensio, 2015; González, 2017; Martínez, 2014). Si además se añade la, ya de por sí, identificación como diferentes en entornos de escasa población, hace que las personas con NEE, y en especial las personas con discapacidad, supongan un objetivo o un sujeto diana para ser víctimas de acoso. 
Por otra parte, el rendimiento escolar se determina por medio de evaluaciones donde el estudiante expone sus conocimientos, siendo dicha evaluación una de las herramientas más utilizadas para valorar su desempeño. Esto adquiere especial relevancia en la actualidad si enmarcamos la evaluación en un modelo basado en la inclusión (Botello, 2016; Castro, Paternina, \& Gutiérrez, 2014). Los estudiantes con necesidades educativas especiales se ven condicionados en un alto porcentaje de casos por su condición de discapacidad, además de factores externos como los apoyos, la disposición de recursos o el interés familiar por las tareas académicas (Pesci, 2015). Si esto es trasladado a entornos rurales donde en muchos casos las condiciones familiares y de recursos materiales y tecnológicos es más escasa que en zonas urbanas, hace que la situación adquiera menores indicadores de éxito (Espada, Gallego \& González, 2019). Es más, si estas variables se unen a las ya mencionadas situaciones de acoso y la falta de recursos para su identificación y su puesta en conocimiento de los profesores y las familias, la brecha se acrecienta en mayor medida. De esta manera, el ambiente escolar, familiar y social donde se desenvuelven los estudiantes con discapacidad influye directamente en su socialización y en su desempeño académico (Musalem \& Castro, 2015).

En lo que respecta al acoso escolar en estudiantes con NEE, diversos estudios aseguran que estos alumnos corren mayor peligro y presentan en un alto porcentaje el hostigamiento e intimidación por parte de los alumnos sin NEE (Vargas \& Paternina, 2017; Monjas, Antón, Francisco, \& Sanchiz, 2014). Incluso en algunos trabajos, las personas con discapacidad han sido consideradas potenciales acosadores debido a la falta de compresión de ciertas convenciones sociales o a la identificación de emociones o situaciones complejas (Ayala, 2015; Enríquez \& Garzón, 2015).

Con respecto a las escuelas que acogen a personas en riesgo de exclusión, algunos trabajos han identificado que las diferencias existentes son mínimas referentes a la cantidad de situaciones de acoso y abusos entre las instituciones públicas y privadas (Santoyo \& Frías, 2014). Sin embargo, y a pesar de la anterior afirmación, los datos muestran que las medidas que se adoptan una vez identificados los casos en las escuelas privadas suelen ser más drásticas que en los centros públicos (Carrasco et al., 2016; Coarite \& Carrillo, 2014).

Por todo ello, el objetivo final de este trabajo ha sido conocer la prevalencia del acoso escolar en estudiantes con necesidades educativas especiales en un entorno rural.

\section{Método}

Este estudio exploratorio ha sido diseñado para conocer un fenómeno y saber cómo se manifiesta, es decir, para llegar a conocer sus propiedades o características (Ibáñez, 2015).

\subsection{Participantes}

La muestra estuvo compuesta por un total de 115 estudiantes correspondientes a séptimo grado de escuelas rurales en la provincia de Machala en El Ecuador. La selección de la muestra fue a través de un muestreo no probabilístico de conveniencia, dado que se tuvo acceso a las aulas donde laboraban parte del equipo de investigación de este trabajo. Entre ellos había estudiantes con NEE, tanto con o sin discapacidad, de los cuales 64 pertenecen a dos escuelas públicas y 51 a dos escuelas particulares de entornos rurales de la provincia de Machala. Un total de 62 varones participaron en el estudio, por lo que el resto, 53 de ellas, fueron mujeres. De estos participantes la media de edad se situó en 10,9 años. Además, 13 de ellos se identificaron con problemas en la lecto-escritura, 5 de ellos con discapacidad intelectual, 3 con déficit de atención e hiperactividad, 2 con autismo, 2 con discapacidad visual, 1 con discalculia y 88 estudiantes restantes que no fueron identificados con ninguna necesidad educativa especial.

Por otro lado, un total de 8 docentes participaron en el estudio. El $100 \%$ de la muestra de docentes fueron mujeres, 4 correspondieron a la escuela pública y 4 a la escuela particular. Todas ellas tenían más de 10 años de experiencia como maestras.

\subsection{Instrumento}

Para evaluar las situaciones de acoso en entornos rurales, un cuestionario ad hoc fue creado bajo el modelo de Marín y Reidl (2013). Este se dividió en cuatro apartados: bullying físico, verbal, psicológico y daños a la propiedad. Las respuestas fueron presentadas en una escala tipo Likert (siempre - a veces 
- rara vez - nunca) con una puntuación de 1 a 4 de manera descendente según el grado de desacuerdo. Dicho instrumento fue aplicado a una pequeña muestra para comprobar su viabilidad. Una vez vista dicha viabilidad y análisis pertinentes sobre su funcionamiento, se realizaron las correcciones sugeridas y se ajustó el cuestionario.

Además, un segundo cuestionario fue desarrollado para identificar aquellas estrategias que utilizaban las docentes para evaluar y trabajar los casos de acoso escolar. Para ello, se diseñó una guía de entrevista a través de un cuestionario ad hoc con seis preguntas con respuestas de opción múltiple. Este instrumento fue validado por un juicio de expertos que evaluó los ítems propuestos. Con las correcciones sugeridas se elaboró el instrumento final para su aplicación.

\subsection{Procedimiento}

Para iniciar la recolección de datos empíricos se entregó a cada una de las autoridades de las instituciones educativas oficios solicitando permiso para aplicar los instrumentos de investigación. Una vez sumillados y aprobados, se procedió a entrevistar a los docentes de séptimo grado (una escuela por día); posteriormente se dialogó con las dos maestras de cada escuela para indagar acerca del número de estudiantes con NEE que tenían en su aula procediendo a seleccionar solo un paralelo.

Se informó a todos los participantes que el cuestionario era voluntario y que disponían de 30 minutos para rellenarlo. Igualmente, se les proporcionó la posibilidad de eliminar los datos una vez analizados, si así lo consideraban oportuno.

\subsection{Análisis de datos}

En primer lugar, los datos fueron tabulados en el programa de análisis estadístico SPSS en su versión 26 (Cronk, 2019). Los estadísticos descriptivos básicos fueron calculados.

\section{Resultados y discusión}

La Tabla 1 muestra el análisis de correlaciones entre las variables de alumnos con NEE y la frecuencia en sufrir acoso escolar para la muestra de estudiantes con diferentes NEE.

Tabla 1.

Resultados de la distribución de frecuencia de doble entrada, con las variables «frecuencia del bullying» $y$ «NEE»

\begin{tabular}{|c|c|c|c|c|c|c|}
\hline & & & \multicolumn{3}{|c|}{ Frecuencia del bullying } & \multirow{2}{*}{ Total } \\
\hline & & & Rara vez & A veces & Siempre & \\
\hline \multirow[t]{14}{*}{ NEE } & \multirow{2}{*}{ Lecto-escritura } & Recuento & 7 & 3 & 3 & 13 \\
\hline & & $\%$ dentro de $\mathrm{B}$. & $63.6 \%$ & $27.3 \%$ & $60.0 \%$ & $48.1 \%$ \\
\hline & \multirow{2}{*}{ Déficit de atención } & Recuento & 0 & 3 & 0 & 3 \\
\hline & & $\%$ dentro de $\mathrm{B}$. & $.0 \%$ & $27.3 \%$ & $.0 \%$ & $11.1 \%$ \\
\hline & \multirow{2}{*}{ Discapacidad intelectual } & Recuento & 1 & 3 & 1 & 5 \\
\hline & & $\%$ dentro de $\mathrm{B}$. & $9.1 \%$ & $27.3 \%$ & $20.0 \%$ & $18.5 \%$ \\
\hline & \multirow[t]{2}{*}{ Discapacidad física } & Recuento & 0 & 1 & 0 & 1 \\
\hline & & $\%$ dentro de $\mathrm{B}$. & $.0 \%$ & $9.1 \%$ & $.0 \%$ & $3.7 \%$ \\
\hline & \multirow[t]{2}{*}{ Discapacidad visual } & Recuento & 1 & 1 & 0 & 2 \\
\hline & & $\%$ dentro de $\mathrm{B}$. & $9.1 \%$ & $9.1 \%$ & $.0 \%$ & $7.4 \%$ \\
\hline & \multirow[t]{2}{*}{ Discalculia } & Recuento & 0 & 0 & 1 & 1 \\
\hline & & $\%$ dentro de $\mathrm{B}$. & $.0 \%$ & $.0 \%$ & $20.0 \%$ & $3.7 \%$ \\
\hline & \multirow[t]{2}{*}{ Autismo } & Recuento & 2 & 0 & 0 & 2 \\
\hline & & $\%$ dentro de $\mathrm{B}$. & $18.2 \%$ & $.0 \%$ & $.0 \%$ & $7.4 \%$ \\
\hline \multirow[t]{2}{*}{ Total } & & Recuento & 11 & 11 & 5 & 27 \\
\hline & & $\%$ dentro de $\mathrm{B}$. & $100.0 \%$ & $\begin{array}{l}100 \\
.0 \%\end{array}$ & $100.0 \%$ & $100.0 \%$ \\
\hline
\end{tabular}

Fuente: elaboración propia. 
Un dato que se muestra y es interesante destacar es que las personas con trastornos en el cálculo, que tradicionalmente no se ha considerado como una necesidad educativa especial, es la categoría que menos acoso sufre dentro de todo el colectivo de NEE. Además, los trastornos de la lecto-escritura aparecen como segunda población con menos casos identificados de acoso en las aulas. Es decir, parece que los estudiantes con dificultades del aprendizaje (en muchos casos denominados con discapacidades invisibles) son el colectivo dentro de las NEE que menos situaciones de bullying están sufriendo en las aulas ordinarias. Igualmente ocurriría con la categoría de estudiantes con trastorno de déficit de atención e hiperactividad, que se sitúan como el tercer grupo con menos casos identificados de bullying. En este sentido, parece evidente que este tipo de necesidades no suponen, por tanto, un foco de atención por parte de los acosadores (Mañanas, González, \& Cortés, 2020). Lo que es significativo de la necesidad de identificar estas variables en los programas de prevención del acoso para intentar frenar esta situación de desventaja por parte del colectivo de personas con necesidades educativas especiales.

Sin embargo, en el sentido opuesto, son las personas con trastornos del espectro del autismo, seguidas de los estudiantes con discapacidad intelectual, quienes señalan en mayor número de ocasiones que han sido víctimas de acoso escolar. Hay que reconocer que las personas con discapacidad son, potencialmente, estudiantes con más probabilidades de sufrir acoso en los entornos educativos (Falla \& Ortega-Ruiz, 2019).

En medio de los dos extremos, se han encontrado las personas con discapacidades sensoriales, en este caso, estudiantes con discapacidad visual, que reportan números que oscilan entre los dos grandes grupos descritos anteriormente. En realidad, en este sentido, las personas con discapacidades sensoriales son, probablemente, el colectivo dentro de la discapacidad que más visibilidad en las etapas educativas ha tenido y, por tanto, existe una mayor normalización de su presencia en las aulas (Pais y Salgado, 2020; Del Barrio, \& Van der Meulen, 2016). Esta situación hace que no sean el principal colectivo diana para sufrir acoso, aunque, evidentemente, no están exentos de esta situación y reportan datos de prevalencia superiores a la media de estudiantes sin discapacidad.

Por otro lado, entre los datos obtenidos a través de las entrevistas a los docentes para identificar las estrategias que se llevaron a cabo para intentar paliar las situaciones de acoso. La muestra total de maestros de escuelas particulares arrojó unos resultados que mostraron la no participación en ningún tipo de actividad formativa respecto al acoso escolar en los últimos 5 años. Por otro lado, sin embargo, el 50\% de los docentes de las escuelas públicas habían asistido a un total de entre 1 y 2 talleres, el 25\% a 3-4 talleres y el 25\% restante no había asistido a ningún taller.

Otro dato interesante resultó sobre el número de medidas que los docentes habían tomado para prevenir el bullying en sus centros. El 100\% de los maestros de las escuelas particulares rara vez habían tomado medidas que previnieran el bullying en el salón de clase; mientras que el $75 \%$ de los docentes de las escuelas públicas algunas veces sí lo habían hecho, en comparación al 25\% que describían una elevada frecuencia en la toma de medidas de prevención frente al acoso.

También cabe destacar el resultado obtenido sobre la implicación que las autoridades educativas de los centros tenían frente al acoso. La totalidad de la muestra para centros particulares describió que las autoridades de las instituciones no se habían interesado por prevenir el acoso a través de ningún tipo de medida, mientras que $50 \%$ de los docentes de las escuelas públicas manifestaron que sí existía en sus centros dicha disposición.

En referencia a los docentes de escuelas particulares, se apreció que el 100\% de los maestros manifiestan que rara vez han conversado con los padres de familia de los niños agresores para darles a conocer el problema; mientras que el $50 \%$ de los maestros de las escuelas públicas afirman que algunas veces lo han realizado, el 25\% rara vez y el $25 \%$ restante muy frecuentemente. Además, también el 100\% de los maestros del mismo tipo de escuelas manifiestan que rara vez han informado a los padres de familia sobre aquellos alumnos que han sido víctimas de acoso infantil o han sido espectadores del acto de acoso, mientras que el $75 \%$ de los maestros de las escuelas públicas afirman que en alguna ocasión sí lo han comunicado, aunque no en todas y el 25\% lo describen como una práctica habitual.

Además, son llamativos los datos que se han identificado sobre la falta de trabajo de formación, de comunicación, de identificación y de resolución de conflictos en las escuelas particulares. Algunos autores (Luna, 2015) ya avanzaron situaciones similares en trabajos previos. Sin embargo, a los autores de este estudio les llama poderosamente la atención que aun teniendo mejores condiciones para poder identificar las situaciones de acoso (menos número de estudiantes con necesidades educativas 
especiales y menor número, en general, de alumnos por salones clase) estas situaciones todavía estén ocurriendo en centros que promueven una mayor atención individualizada. Esto es algo que además va en contra de otros trabajos publicados que promueven buenas prácticas educativas en sentidos completamente opuestos a esta visión (Sánchez \& Martín, 2019).

\section{Conclusiones}

Después de analizar y discutir los resultados hallados, los autores de este trabajo identifican algunas cuestiones de vital importancia para la obtención de una educación inclusiva de calidad (Sánchez \& Díez, 2013). De hecho, si se tienen en cuenta los Objetivos de Desarrollo Sostenible, más concretamente, el objetivo 4, relacionado con la educación inclusiva, en él se indica que dicha educación debe ser inclusiva sí, pero de calidad también. Evidentemente no se puede llegar a una educación inclusiva de calidad si no se atiende a las necesidades de colectivos tan vulnerables como son las personas con discapacidad (González-Montesino \& Espada, 2020) y, en especial, las personas con discapacidad intelectual y del desarrollo y las personas con autismo. Además, como se ha venido afirmando a lo largo de este trabajo, esta situación es de especial relevancia cuando sucede en entornos rurales con menos recursos y, en líneas generales, más reacios a un cambio de modelo donde la diversidad tiene cabida.

Además, no solo los grupos mencionados anteriormente son vulnerables, aquellos estudiantes que conviven con una necesidad educativa menos visible, en muchos casos, son menos atendidos por los profesionales debido a su invisibilización. Esto es una gran tarea por desarrollar y es que en menor medida se pone el foco en la literatura académica en estos grupos poblacionales frente a situaciones de acoso.

De hecho, en la actualidad la gran mayoría de trabajos que estudian el acoso escolar o los programas que intentan prevenirlo, no están teniendo en cuenta la variable de la discapacidad. En realidad, se analiza como un factor más en las probabilidades de sufrir acoso, pero no se tiene en cuenta como personas que están en las aulas sufriendo en la mayor parte de casos acoso en un porcentaje muy superior al resto de compañeros en los salones clase. Por lo tanto, los autores de este trabajo creen necesario que publicaciones como esta que arrojan datos evidentes de la situación en la que se está produciendo el acoso en las aulas respecto de estos colectivos vulnerables, son necesarias para que se puedan adoptar las medidas oportunas y que las autoridades pertinentes puedan obtener una base empírica en la que sustentar sus decisiones al respecto.

Por otro lado, también creemos fundamental destacar que los datos obtenidos acerca de cómo se está interviniendo y cómo se está actuando en los centros en función de su titularidad, muestran datos alarmantes que se deberían tener en cuenta con la máxima premura posible. Si bien es cierto que los gobiernos deben garantizar una educación para todos sus ciudadanos y que esto se lleva a cabo a través de las escuelas públicas, no puede existir esa descompensación frente a los centros particulares que parece que no identifican o no quieren identificar el acoso escolar en sus centros. Esto se puede deber a numerosas cuestiones que los autores prefieren no aventurar, pero sí se cree de primera necesidad realizar más estudios al respecto contrastando algunos datos para observar si este trabajo ha supuesto un fenómeno aislado o, realmente, es una situación que se está dando en la mayor parte de centros particulares del país.

Por último, y en la misma línea de lo expuesto anteriormente, los autores somos conscientes de las limitaciones que este estudio puede tener. Sería recomendable explorar nuevas regiones y ampliar la muestra para poder extrapolar los resultados al país entero y que los datos puedan ser inferenciales de todos los centros, tanto públicos como particulares.

\section{Referencias}

Abadio, W., Lossi, M., Carvalho, F., Lopes, D., Yoshinaga, A., \& Carvalho, D. (2015). Causas del bullying: resultados de la Investigación Nacional de la Salud del Escolar. Revista LatinoAmericana de Enfermagem, 23(2), pp. 1-8. doi: 10.1590/0104-1169.0022.2552.

Amaya, C. (2016). Los imaginarios sociales, el pensamiento crítico y la no violencia, una forma para enfrentar el acoso escolar. Estudios pedagógicos, 27(3), pp. 27-48. 42(3), pp. 27-48. doi: $10.4067 /$ S0718-07052016000400002. 
Arias, W. (2014). ¿Qué es el bullying?: actores, causas e intervención. Revista de Psicología de Arequipa, 4(1), pp. 11-32.

Aristimuño, A., \& Noya, J. C. (2015). La convivencia escolar y el fenómeno del Bullying en la enseñanza secundaria de Uruguay. Un estudio de caso. Páginas de Educación, 8(2), pp. 1-29. doi: doi.org/10.22235/pe.v8i2.691.

Asanza, M. I., Pilco, M., \& Alvarado, P. (2015). El Bullying en la universidad. Revista Atlante: Cuadernos de Educación y Desarrollo. 4(1), pp. 11-13.

Ayala, M. D. (2015). Violencia escolar: Un problema complejo. Ra Ximbai, 11(4), pp. 493-509. https://www.redalyc.org/pdf/461/46142596036.pdf. doi: 10.35197/rx.11.01.e2.2015.36.ma.

Botello, H. A. (2016). Efecto del acoso escolar en el desempeño lector en Colombia. Zona próxima. Revista del Instituto de Estudios en Educación Universidad del Norte, 24, pp. 1-12. doi: dx.doi.org/10.14482/zp.24.720.

Carrasco, C. L., Verdejo, T., Asunr, D., Álvarez, J. P., Bustos, C., Ortiz, S., ... Valdivia, N. (2016). Concepciones de infancia en una escuela con altos índices de violencia escolar de estudiantes a profesores. Revista Latinoamericana de Ciencias Sociales, Niñezy Juventud, 14(2), pp. 1145-1159. doi: 10.11600/1692715x.14218210814.

Castro, S., Paternina, A., \& Gutiérrez, M. (2014). Factores pedagógicos relacionados con el rendimiento académico en estudiantes de cinco instituciones educativas del distrito de Santa Marta, Colombia. Revista Intercontinental de Psicologia y Educación, 16(2), pp. 151-169. https://www.redalyc.org/pdf/802/80231541009.pdf.

Coarite, E., \& Carrillo, G. (2014). Consideraciones sobre la violencia escolar y los conflictos de estudiantes. Revista de Investigación Educativa, 7(2), pp. 175-186. http://www.scielo.org.bo/pdf/rieiii/v7n2/v7n2 a11.pdf.

Cobián, C., Nizama, A., Ramos, D., \& Mayta, P. (2015). Medición y magnitud del bullying en Perú. Revista Peruana de Medicina Experimental y Salud Pública, 32(1), pp. 191-204. http://www.scielo.org.pe/pdf/rins/v32n1/a32v32n1.pdf. doi: 10.17843/rpmesp.2015.321.1598.

Córdova, A. J., Velázquez, M., \& Silva, E. (2015). Características de bullying entre jóvenes estudiantes de secundaria usuarios y no usuarios de drogas. Revista Intercontinental de Psicología y Educación, 17(2), pp. 103-120. https://www.redalyc.org/articulo.oa?id=80247939006.

Cronk, B. C. (2019). How to use SPSS®: A step-by-step guide to analysis and interpretation. Routledge. doi: $10.4324 / 9780429340321$.

Del Barrio, C., \& van der Meulen, K. (2016). Maltrato por abuso de poder entre iguales en el alumnado con discapacidad. Pensamiento Psicológico, 14(1), pp. 103-118. doi: 10.11144/Javerianacali.PPSI14-1.mapi.

Enríquez, M. F., \& Garzón, F. (2015). El acoso escolar. Saber, Ciencia y Libertad, 10(1), pp. 219-233. doi: $10.18041 / 2382-3240 /$ saber.2015v10n1.983.

Erazo, O. (2016). Identificación y descripción de la intimidación escolar en instituciones educativas del municipio de Popayán. Diversitas: Perspectivas en Psicologia, 12(1), pp. 55-72. doi: 10.15332/s17949998.2016.0001.04.

Espada, R. M., Gallego, M., \& González-Montesino, R. H. (2019). Diseño Universal del Aprendizaje e inclusión en la Educación Básica. Alteridad, 14(2), pp. 207-218. doi: 10.17163/alt.v14n2.2019.05.

Falla, D., \& Ortega-Ruiz, R. (2019). Los Escolares Diagnosticados con Trastorno del Espectro Autista y Víctimas de Acoso Escolar: una Revisión Sistemática. Educational Psychology, 25(2), pp. 7790. doi: 10.5093/psed2019a6.

Garaigordobil, M. (2015). Ciberbullying en adolescentes y jóvenes del País Vasco: Cambios con la edad. Anales de Psicología, 31(3), pp. 1069-1076. doi: 10.6018/analesps.31.3.179151.

Garaigordobil, M., Martínez, V., \& Páez, D. (2015). Bullying y cyberbulling: diferencias entre colegios públicos-privados y religiosos-laicos. Pensamiento Psicológico, 13(1), pp. 39-52. https://revistas.javerianacali.edu.co/index.php/pensamientopsicologico/article/view/935. doi: 10.11144/Javerianacali.PPSI13-1.bcdc.

García, M., \& Ascensio, C. (2015). Bullying y violencia escolar: diferencias, similitudes, actores, consecuencias y origen. Revista Intercontinental de Psicologia y Educación, 17(2), pp. 9-38. https://dialnet.unirioja.es/servlet/articulo? codigo $=6532205$.

González, A. (2017). Acoso Escolar y Necesidades Educativas Especiales. Tesis Doctoral, Universidad de Extremadura. 
González-Montesino, R. H., \& Espada, R. M. (2020). Espacios universitarios de aprendizaje inclusivos, bilingües y multimodales: el caso del alumnado sordo signante. Revista De Estilos De Aprendizaje, 13(25), pp. 70-83. http://revistaestilosdeaprendizaje.com/article/view/1519.

Gómez, A. (2014). Bullying: el poder de la violencia. Una perspectiva cualitativa sobre acosadores y víctimas en primarias de Colima. Consejo Mexicano de Investigación Educativa, 18(58), pp. 839-870. http://www.scielo.org.mx/pdf/rmie/v18n58/v18n58a8.pdf.

Herrera, M., Romera, E., \& Ortega, R. (2018). Bullying y Cyberbullying en Latinoamérica. Un estudio bibliométrico. RMIE. Revista Mexicana de Investigación educativa, 23(76), pp. 125-155. http://www.scielo.org.mx/pdf/rmie/v23n76/1405-6666-rmie-23-76-125.pdf.

Ibáñez, J. (2015). Métodos, técnicas e instrumentos de la investigación criminológica. Madrid: DYKINSON.

Lugones, M., \& Ramírez, M. (2017). Bullying: aspectos históricos, culturales y sus consecuencias para la salud. Revista Cubana de Medicina General Integral, 33(1), pp. 154-162. http://revmgi.sld.cu/index.php/mgi/article/view/277/132.

Luna, E. A. (2015). Estudio epidemiológico de la violencia y acoso escolar (bullying): situación actual en la ciudad de Trujillo-2014. Pueblo continente, 25(1), pp. 209-219. http://journal.upao.edu.pe/PuebloContinente/article/view/92.

Mañanas, M., González, B., \& Cortés, P. (2020). Historias de vida de personas con discapacidad intelectual: Entre el acoso y exclusión en la escuela como moduladores de la identidad. Revista Educación, Politica y Sociedad, 5(1), pp. 60-84 https://dialnet.unirioja.es/servlet/articulo?codigo $=7186980$.

Marín, A., \& Reidl, L. M. (2013). Validación psicométrica del cuestionario «Así nos llevamos en la escuela» para evaluar el hostigamiento escolar (bullying) en primarias. Revista mexicana de investigación educativa, 18(56), pp. 11-36. http://www.scielo.org.mx/pdf/rmie/v18n56/v18n56a2.pdf.

Martínez, S. (2014). Bullying: violencia en la escuela. Revista Digital Universitaria, 15(1). http://www.revista.unam.mx/vol.15/num $1 / \operatorname{art} 02 /$.

Monjas, M. I., Antón, L., Francisco, G., \& Sanchiz, M. L. (2014). Rechazo y victimización al alumnado con necesidad de apoyo educativo en primero de primaria. Anales de Psicología, 30(2), pp. 499-511. doi: 10.6018/analesps.30.2.158211.

Moreno-Rodríguez, R., López, J. L., Carnicero, J. D., Garrote, I., \& Sánchez, S. (2017). Teachers' Perception on the Inclusion of Students with Disabilities in the Regular Education Classroom in Ecuador. Journal of Education and Training Studies, 5(9), pp. 45-53. doi: 10.11114/jets.v5i9.2573.

Musalem, R., \& Castro, P. (2015). Qué se sabe de bullying. Revista Médica Clinica Las Condes, 26(1), pp. 14-23. $\quad$ https://www.elsevier.es/es-revista-revista-medica-clinica-las-condes-202-pdfS071686401500005X. doi: 10.1016/j.rmclc.2014.12.002.

Pais, M. E. M., \& Salgado, F. R. (2020). La atención a la diversidad en el aula: dificultades y necesidades del profesorado de educación secundaria y universidad. Contextos Educativos. Revista de Educación, 25, pp. 257-274. doi: 10.18172/con.3734.

Pesci, A. L. (2015). Prevención del bullying en México: El caso de los niños y adolescentes sobredotados. Revista de El Colegio de San Luis, 5(10), pp. 104-133. http://www.scielo.org.mx/scielo.php?script=sci_arttext\&pid=S1665-899X2015000200104. doi: $10.21696 / \mathrm{rcsl} 5102015625$.

Ruiz, R., Riuró, M., \& Tesouro, M. (2015). Estudio del bullying en el ciclo superior de primaria. Educación XX1, 18(1), pp. 345-368. doi: 10.5944/educxx1.18.1.12384.

Sánchez, S., \& Díez, E. (2013). La educación inclusiva desde el currículum: el Diseño Universal para el Aprendizaje. En: TRANSFORMANDO LA ESCUELA: Educación inclusiva, equidad y derecho a la diferencia, pp. 107-119. Wolters Kluwer.

Sánchez, S., \& Martín, R. A. (2019). Buenas prácticas en la educación superior basadas en el diseño universal como marco de referencia. En ¿Avanzamos hacia universidades más inclusivas?: de la retórica a los hechos, pp. 121-137. Dykinson. doi: 10.2307/j.ctvr7f5rq.10.

Santoyo, D., \& Frías, S. M. (2014). Acoso escolar en México: actores involucrados y sus características. Revista Latinoamericana de Estudios Educativos (México), 34(4), pp. 13-41. https://dialnet.unirioja.es/servlet/articulo? codigo $=6015945$.

Vargas, D., \& Paternina, Y. (2017). Relación entre habilidades sociales y acoso escolar. Cultura. Educación y Sociedad, 8(2), pp. 61-78. doi: 10.17981/cultedusoc.8.2.2017.05. 
Vergel, M., Martínez, J., \& Zafra, S. (2016). Factores asociados al bullying en instituciones de educación superior. Revista Criminalidad, 58(2), pp. 197-208. http://www.scielo.org.co/pdf/crim/v58n2/v58n2a08.pdf. 
Anexo A

AÑO DE BÁSICA:

EDAD:

GÉNERO: M ( ) F ( )

PRESENTA DISCAPACIDAD: SÍ ( ) NO ( ) ¿CUÁL?:

FECHA DE LA APLICACIÓN: AÑO / ........MES:....... DÍA/.......

\begin{tabular}{|c|c|c|c|c|}
\hline TIPOS DE BULLYING & $\begin{array}{l}\text { (4) } \\
\text { SIEMPRE }\end{array}$ & $\begin{array}{l}\text { (3) } \\
\text { A VECES }\end{array}$ & $\begin{array}{l}\text { (2) } \\
\text { RARA } \\
\text { VEZ }\end{array}$ & $\begin{array}{l}\text { (1) } \\
\text { NUNCA }\end{array}$ \\
\hline \multicolumn{5}{|l|}{ Físico } \\
\hline - Te has peleado con tus compañeros & & & & \\
\hline - Te han pegado tus compañeros & & & & \\
\hline - Te han dado puñetazos tus compañeros & & & & \\
\hline - Te han empujado tus compañeros & & & & \\
\hline - Te han dado patadas tus compañeros & & & & \\
\hline \multicolumn{5}{|l|}{ Verbal } \\
\hline - Te han gritado tus compañeros & & & & \\
\hline - Te han insultado tus compañeros & & & & \\
\hline - Te han dicho groserías tus compañeros & & & & \\
\hline - Se te han burlado tus compañeros & & & & \\
\hline - Te han puesto apodos tus compañeros & & & & \\
\hline \multicolumn{5}{|l|}{ Psicológico } \\
\hline - Te han discriminado tus compañeros & & & & \\
\hline - Te han amenazado tus compañeros & & & & \\
\hline - Te han ignorado tus compañeros & & & & \\
\hline - Te han criticado tus compañeros & & & & \\
\hline $\begin{array}{l}\text { - Te han echado la culpa sin razón tus } \\
\text { compañeros }\end{array}$ & & & & \\
\hline Daño a la propiedad & & & & \\
\hline - Te han robado las cosas tus compañeros & & & & \\
\hline $\begin{array}{l}\text { - Te han escondido las cosas tus compa- } \\
\text { ñeros }\end{array}$ & & & & \\
\hline $\begin{array}{l}\text { - Te han quitado las cosas tus compañe- } \\
\text { ros }\end{array}$ & & & & \\
\hline $\begin{array}{l}\text { - Te han interrumpido tus compañeros } \\
\text { mientras haces tu tarea }\end{array}$ & & & & \\
\hline - Te han roto las cosas tus compañeros & & & & \\
\hline
\end{tabular}

\section{CUESTIONARIO DIRIGIDO A LOS DOCENTES}

Tema de investigación:

Comparar la incidencia del bullying en el rendimiento académico de los estudiantes con o sin NEE de escuelas públicas y privadas

Objetivos de la investigación:

- Comprar el nivel de bullying en los estudiantes asociados o no a una NEE entre escuelas públicas y privadas.

- Identificar la correlación entre el bullying y los ámbitos académicos, sociales y emocionales en los estudiantes con NEE frente a los estudiantes sin NEE.

- Identificar las estrategias que utilizan los docentes para erradicar los casos de bullying.

\section{Datos Informativos:}

Género: $\quad M() \quad F()$ 
Grado: ...................

Fecha de la aplicación del cuestionario:

Hora de la aplicación del cuestionario:

\section{Instrucciones:}

El presente cuestionario es parte de una investigación cuyo objetivo es comparar el grado de afectación que tiene el bullying en el rendimiento académico de los estudiantes con NEE frente a los estudiantes regulares; la información que nos proporcione se utilizará para fines de la investigación será estrictamente confidencial, le solicitamos atentamente su cooperación y apoyo contestando a los siguientes cuestionamientos. Gracias

Tome en consideración lo siguiente:

- Marque con una «X» la opción de su preferencia.

- Debe expresar su respuesta tomando en consideración los parámetros de cada pregunta.

- Leer totalmente la pregunta antes de contestar.

- Contestar cada una de las preguntas.

- Por favor, no usar correctores ni borradores, tampoco manchar la hoja.

- No se permite contestar más de una vez en cada pregunta.

- $\quad$ El presente cuestionario es totalmente anónimo.

- Tiene 15 minutos para contestar las preguntas.

\section{Preguntas:}

1. ¿A cuántos talleres, cursos, seminarios o capacitaciones, usted ha asistido durante los cinco últimos años acerca del bullying?
( ) 0
( ) 1 - 2
( ) $3-4$
( ) $>4$

Y si no ha asistido, ¿por qué?

2. ¿Con qué frecuencia usted ha tomado medidas que prevengan el bullying en el salón de clase?

( ) Muy frecuentemente

( ) Frecuentemente

( ) Algunas veces

( ) Rara vez

( ) Nunca

¿Por qué?

3. ¿Las autoridades de la institución educativa donde usted labora se ha interesado por prevenir el bullying?

( ) Sí

( ) $\mathrm{No}$

Si la respuesta es negativa, ¿cuál es el motivo? 
4. ¿Con qué frecuencia usted ha conversado con los padres de familia de los niños agresores para darles a conocer el problema?

( ) Muy frecuentemente

( ) Frecuentemente

( ) Algunas veces

( ) Rara vez

( ) Nunca

¿Por qué?

5. ¿Con qué frecuencia usted ha conversado con los padres de familia de los niños que han sido víctimas de acoso infantil o bullying para darles a conocer el problema?

( ) Muy frecuentemente

( ) Frecuentemente

( ) Algunas veces

( ) Rara vez

( ) Nunca

¿Por qué?

6. ¿Con qué frecuencia usted ha conversado con los padres de familia de los niños espectadores del acoso infantil o bullying para darles a conocer el problema?

( ) Muy frecuentemente

( ) Frecuentemente

( ) Algunas veces

( ) Rara vez

( ) Nunca

¿Por qué?

Observaciones:

Fecha: 